\title{
Evaluation of Coronary Calcium Score by Multidetector Computed Tomography in Relation to Endothelial Function and Inflammatory Markers in Asymptomatic Individuals
}

\author{
Mahmoud M. Ramadan, MD*,**; Essam M. Mahfouz, MD**; Gamal F. Gomaa, MD**; \\ Tarek A. El-Diasty, MD ${ }^{\dagger}$; Louie Alldawi, MD*; Taruna Ikrar, MD*; \\ Ding Limin, MD*; Makoto Kodama, MD*; Yoshifusa Aizawa, MD*
}

\begin{abstract}
Background Coronary calcification has been correlated with the presence and extent of coronary artery disease (CAD), so in the present study the associations between coronary artery calcification score (CACS) and endothelial dysfunction, as well as the important inflammatory markers C-reactive protein (CRP), interleukin (IL)-6, and oxidized low-density lipoprotein (OxLDL), were studied in asymptomatic individuals at intermediate risk for CAD.

Methods and Results The study group comprised 177 subjects (103 males) aged 50.6 \pm 5.9 years. CACS was measured by multidetector computed tomography using the Agatston method. Endothelium-dependent flowmediated dilatation (FMD) and endothelium-independent nitroglycerin-mediated dilatation (NMD) were measured by high-resolution external brachial ultrasound. Coronary artery calcification (CAC) was detected in 82 subjects (52 males), and the median CACS was 143 [31-311.25] units. After adjusting for gender and body mass index, $\log (\mathrm{CACS}+1)$ correlated positively with age $(\mathrm{r}=0.401, \mathrm{p}<0.001)$ and IL-6 levels $(\mathrm{r}=0.442, \mathrm{p}<0.001)$, and negatively with FMD $(r=-0.511, \mathrm{p}<0.001)$. The correlations of $\log (\mathrm{CACS}+1)$ with $\mathrm{CRP}$ and OxLDL levels, and with NMD, were non-significant. In a multivariate-adjusted logistic regression model, age (odds ratio $(\mathrm{OR})=$ 1.083 [1.014-1.156]), serum IL-6 level (OR=3.837 [2.166-6.798]) and FMD (OR=0.851 [0.793-0.913]) were significantly and independently associated with CAC.

Conclusions Peripheral endothelial function inversely correlated with CACS, whereas IL-6 level was associated with CACS. Testing for endothelial function and IL-6 level may improve cardiovascular risk assessment and help target the therapeutic strategies in asymptomatic patients at intermediate CAD risk. (Circ J 2008; 72: $778-785)$
\end{abstract}

Key Words: C-reactive protein; Coronary calcification; Endothelial dysfunction; Interleukin-6; Oxidized lowdensity lipoprotein

C oronary artery calcification (CAC) is an anatomic disease marker that has been correlated with the presence and extent of coronary artery disease (CAD)! Fortunately, noninvasive detection of CAC and quantitative estimation of its score (CACS) can be done by multidetector computed tomography (CT).

Endothelial dysfunction is now recognized as a key early event in atherogenesis, because it is linked to CAD risk ${ }^{3}$ and future adverse cardiovascular events. It is now possible to indirectly test endothelial function by means of endotheliumdependent flow-mediated dilatation (FMD) and endothe-

(Received August 13, 2007; revised manuscript received December 3, 2007; accepted December 13, 2007)

*Division of Cardiology, First Department of Internal Medicine, Niigata University Graduate School of Medical and Dental Sciences, Niigata, Japan, **Department of Cardiology, Mansoura Faculty of Medicine and Department of Radiology, Mansoura Urology and Nephrology Center, Mansoura University, Mansoura, Egypt

Mailing address: Mahmoud M. Ramadan, MD, Division of Cardiology, Niigata University Hospital, 1-754 Asahimachi Dori, Niigata 9518510, Japan. E-mail: amamod@med.niiagata-u.ac.jp

All rights are reserved to the Japanese Circulation Society. For permissions, please e-mail: cj@j-circ.or.jp lium-independent nitroglycerin-mediated dilatation (NMD) of the brachial artery via external brachial ultrasound. However, only 2 previous studies ${ }^{5,6}$ have investigated the relationship between the extent of CAC and endothelial function in subjects along the whole risk spectrum for $\mathrm{CAD}$, and they reported conflicting results. Therefore, in this study we tested the hypothesis that CACS is associated with endothelial dysfunction as reflected by FMD and NMD of the brachial artery, focusing on asymptomatic subjects at intermediate risk for $\mathrm{CAD}$, which is the category of patients of particular interest for the purposes of primary prevention and risk identification and reduction.

Both C-reactive protein (CRP) and interleukin-6 (IL-6) are closely associated with CAD pathogenesis 7,8 Similarly, oxidized low-density lipoprotein (OxLDL) is believed to play a key role in the development of atherosclerosis? However, studies of CAC in relation to inflammatory markers (other than CRP) are generally scarce. To test whether important inflammatory markers, such as CRP, IL-6, and OxLDL, are related to coronary atherosclerotic burden (as defined by CACS) beyond established risk factors in asymptomatic individuals at intermediate risk for CAD, we sought to determine the benefit of including information on 
endothelial function and inflammatory markers in the current models of CAD risk assessment, prevention, and treatment used for this category of patients.

\section{Methods}

\section{Study Design and Participants}

From January, 2002 to February, 2004, we enrolled 177 asymptomatic, ethnically Middle Eastern Egyptians of both genders who were in the intermediate-risk category according to the 10-year Framingham Study risk score (FRS) for $\mathrm{CAD}$ events. We used the risk factor information to calculate the risk of coronary events during a 10 -year period according to the FRS calculation algorithm ${ }^{10}$ and included only those with a 10-20\% risk of developing CAD.

Subjects aged 40-59 years with at least 1 coronary risk factor were recruited from an internal database comprising more than 6,000 outpatients of Mansoura University Hospital, Mansoura, Egypt, who were asymptomatic for CAD. Initially, 965 subjects were invited to participate in our study by letter (another was sent in cases of non-response) and telephone calls (whenever possible). Agreement to participate was received from 786 subjects, 48 of whom later withdrew. Thus, 738 subjects aged $40-59$ years with at least 1 coronary risk factor remained and underwent the preliminary evaluation. Subjects were also excluded if they had a history of ischemic heart disease.

All subjects were interviewed to complete a detailed health questionnaire. Family history of CAD was pertinent if established CAD was present in a first-degree male relative aged $\leq 55$ years or female relative aged $\leq 65$ years of age. Current or past history of smoking was relevant if the subject had smoked $>10$ cigarettes/day for at least 1 year. Hypertension was defined as systolic pressure $\geq 140 \mathrm{mmHg}$, diastolic pressure $\geq 90 \mathrm{mmHg}$, or current use of antihypertensive medication. Diabetes was defined as fasting glucose $>125 \mathrm{mg} / \mathrm{dl}$ (if untreated) or previous physician diagnosis and treatment with diet, oral hypoglycemic drugs, or insulin. Body mass index was calculated as weight $/ \mathrm{height}^{2}\left(\mathrm{~kg} / \mathrm{m}^{2}\right)$; and obesity was defined as body mass index $\geq 30 \mathrm{~kg} / \mathrm{m}^{2}$. Hypercholesterolemia was defined as a total cholesterol level $>200 \mathrm{mg} / \mathrm{dl}$ or the use of cholesterol-lowering medications.

Participants were given appointments to undergo the planned investigations, stressing an overnight fast (including stoppage of all medications) for at least $12 \mathrm{~h}$ prior to testing. Resting systolic and diastolic arterial blood pressures $(\mathrm{ABP})$ were recorded by mercury sphygmomanometer as the average of the last 2 of 3 readings, each 5 min apart with the subject seated. Standard 12-lead electrocardiograms (ECGs) were recorded to evaluate the presence of left ventricular hypertrophy (LVH), which was diagnosed as Cornell voltage duration product $>2,440 \mathrm{mV} / \mathrm{ms}$. Additionally, 2-dimensional echocardiographic examination was preformed and the left ventricular mass $(\mathrm{g})$ was calculated according to the method recommended by the American Society of Echocardiography [LV mass $=0.8(1.04[\mathrm{LVDd}+$ $\left.\mathrm{IVST}+\mathrm{PWT}]^{3}-\mathrm{LVDd}^{3}\right)+0.6$, where $\mathrm{LVDd}=$ left ventricular diastolic dimension $(\mathrm{mm})$, IVST =interventricular septum thickness $(\mathrm{mm})$, and PWT=posterior wall thickness $(\mathrm{mm})]$ followed by indexing to body surface area $\left(\mathrm{m}^{2}\right)$.

The study protocol was reviewed and accepted by the local Ethical Review Board of the Mansoura University Faculty of Medicine, and written informed consent was given by each participant.

\section{Laboratory Methods}

On the day of screening after a 12-h fast, antecubital venous blood samples were drawn into vacuum tubes with appropriate additives for assessment of glucose, lipids, liver function, complete blood count, serum electrolytes, serum creatinine, and uric acid. Serum high-sensitive CRP was measured by particle-enhanced immunoturbidometric latex agglutination assay (Dade Behring, Newark, DE, USA) and IL-6 was measured by high-sensitivity immunoassay (Quantikine human IL-6, R\&D Systems, Minneapolis, MN, USA). OxLDL was measured by a sensitive ELISA method specific for detecting malondialdehyde-modified LDL!1

\section{Endothelial Measurements}

FMD of the brachial artery following transient ischemia is a noninvasive method of assessing endothelial function, which we performed according to the guidelines described by Corretti et al! ${ }^{2}$ Following an overnight fast, and approximately $1 \mathrm{~h}$ after the CT scanning was completed, the subject remained supine (although this is not superior to the seated position ${ }^{13}$ ) while the right brachial artery was scanned in the longitudinal section 3-5 cm above the antecubital fossa. Brachial artery diameter was measured on B-mode ultrasound images using a 7.0-MHz linear-array transducer and a standard Acuson 128 XP/10 system (Mountain View, CA, USA). After recording baseline measurements, the cuff was placed proximal to the scanned section of brachial artery and inflated to $\geq 50 \mathrm{mmHg}$ above the subject's systolic ABP for $5 \mathrm{~min}$ to create forearm ischemia. Subsequently, the cuff was deflated and the arterial diameter was measured $60 \mathrm{~s}$ after deflation. Endothelium-dependent dilatation was expressed as the percentage change in brachial artery diameter from baseline to reactive hyperemia.

Endothelium-independent dilatation was assessed by measuring changes in brachial artery diameter after sublingual nitroglycerin administration. Following endotheliumdependent measurements for $15 \mathrm{~min}$, baseline resting images were again taken, followed by sublingual administration of $0.5 \mathrm{mg}$ nitroglycerin; measurements were repeated $3 \mathrm{~min}$ later. Endothelium-independent dilatation was expressed as the percentage change in brachial artery diameter from baseline to that following sublingual nitroglycerin administration.

\section{Coronary Calcium Imaging and Quantification}

CAC scanning was performed using an 8-slice multidetector-row CT scanner (Light Speed Plus CT scanner, General Electric Medical Systems, Milwaukee, WI, USA) with prospective ECG gating using sequential data acquisition. Scans were prospectively initiated at $50 \%$ of the RR interval. CT scanning was postponed if the resting pulse rate exceeded 75 beats $/ \mathrm{min}$, in which case controlling the pulse rate with intravenous $\beta$-blocker was attempted and the scan was performed 20 min later if this was successful. Forty-eight consecutive non-overlapping $2.5 \mathrm{~mm}$-thick slices were acquired, with simultaneous acquisition of 8 slices $(140 \mathrm{kV}, 150 \mathrm{~mA}$, gantry rotation time $500 \mathrm{~ms}$, and temporal resolution $330 \mathrm{~ms}$ ). All sections were reviewed by 2 experienced radiologists who were unaware of all clinical data. Image review and CAC scoring were done at a special Advantage Window 4.1 workstation (General Electric Medical Systems) using SmartScore software.

CACS was quantified by the method of Agatston et al ${ }^{14}$ in which CAC is defined as an area of at least 2 contiguous 
Table 1 Baseline Characteristics and Comparisons of Subjects With and Without CAC

\begin{tabular}{|c|c|c|c|c|}
\hline & $\begin{array}{c}\text { Total } \\
(n=177)\end{array}$ & $\begin{array}{c}C A C \text {-positive } \\
(n=82)\end{array}$ & $\begin{array}{c}\text { CAC-negative } \\
(n=95)\end{array}$ & $p$ value \\
\hline \multicolumn{5}{|l|}{ History } \\
\hline Age (years) & $50.6 \pm 5.9$ & $52.3 \pm 5.1$ & $48.7 \pm 6.1$ & $<0.001 * *$ \\
\hline \multicolumn{5}{|l|}{ Gender } \\
\hline M & $103(58.2 \%)$ & $52(63.4 \%)$ & $51(53.7 \%)$ & \multirow{2}{*}{0.248} \\
\hline$F$ & $74(41.8 \%)$ & $30(36.6 \%)$ & $44(46.3 \%)$ & \\
\hline \multicolumn{5}{|l|}{ Medications } \\
\hline$A C E I s / A R B s$ & $99(55.9 \%)$ & $45(54.9 \%)$ & $54(56.8 \%)$ & 0.912 \\
\hline Aspirin & $108(61.0 \%)$ & $49(59.8 \%)$ & $59(62.1 \%)$ & 0.869 \\
\hline$\beta$-blockers & $34(19.2 \%)$ & $19(23.2 \%)$ & $15(15.8 \%)$ & 0.293 \\
\hline Calcium-channel blockers & $46(26.0 \%)$ & $20(24.4 \%)$ & $26(27.4 \%)$ & 0.781 \\
\hline Diuretics & $86(48.6 \%)$ & $41(50.0 \%)$ & $45(47.4 \%)$ & 0.843 \\
\hline Statins & $27(15.3 \%)$ & $12(14.6 \%)$ & $15(15.8 \%)$ & 0.997 \\
\hline \multicolumn{5}{|l|}{$C A D$ risk factors } \\
\hline$C A D$ family history & $81(45.8 \%)$ & $40(48.8 \%)$ & $41(43.2 \%)$ & 0.551 \\
\hline Current smoking & $68(38.4 \%)$ & $27(32.9 \%)$ & $41(43.2 \%)$ & 0.215 \\
\hline Diabetes mellitus & $117(66.1 \%)$ & $56(68.3 \%)$ & $61(64.2 \%)$ & 0.681 \\
\hline Hypertension & $108(61.0 \%)$ & $53(64.6 \%)$ & $55(57.9 \%)$ & 0.446 \\
\hline Obesity $\left(B M I \geq 30 \mathrm{~kg} / \mathrm{m}^{2}\right)$ & $79(44.6 \%)$ & $37(45.1 \%)$ & $42(44.2 \%)$ & 0.903 \\
\hline 10-year FRS & $15.2[11.5-17.9]$ & $17.7[11.9-18.4]$ & $14.2[11.0-16.6]$ & $0.045^{*}$ \\
\hline \multicolumn{5}{|c|}{ Anthropometric/medical measurements } \\
\hline Body weight (kg) & $82.3 \pm 15.8$ & $83.2 \pm 13.9$ & $81.6 \pm 17.3$ & 0.527 \\
\hline Height $(\mathrm{cm})$ & $167.0 \pm 8.2$ & $167.7 \pm 7.8$ & $166.4 \pm 8.4$ & 0.284 \\
\hline$B M I\left(k g / m^{2}\right)$ & $29.7 \pm 6.1$ & $29.7 \pm 5.4$ & $29.6 \pm 6.7$ & 0.939 \\
\hline Systolic ABP (mmHg) & 135 [125-155] & 137.5 [125-155] & 135 [125-155] & 0.807 \\
\hline Diastolic ABP (mmHg) & 90 [80-100] & $90[80-100]$ & $90[75-100]$ & 0.579 \\
\hline Mean ABP (mmHg) & 105 [95-117.5] & $104.5[95-117.1]$ & 105 [93.3-118.3] & 0.641 \\
\hline \multicolumn{5}{|l|}{ Laboratory measurements } \\
\hline Fasting glucose $(\mathrm{mg} / \mathrm{dl})$ & $168.0 \pm 53.3$ & $172.0 \pm 49.0$ & $164.0 \pm 56.7$ & 0.321 \\
\hline Hemoglobin Alc (\%) & $6.02 \pm 2.05$ & $6.09 \pm 2.12$ & $5.95 \pm 1.98$ & 0.637 \\
\hline Total cholesterol $(\mathrm{mg} / \mathrm{dl})$ & 196 [166.8-266.2] & 210.5 [169.4-266.5] & $180[162-266]$ & 0.121 \\
\hline HDL-cholesterol $(\mathrm{mg} / \mathrm{dl})$ & $39.4 \pm 8.5$ & $38.6 \pm 8.1$ & $40.1 \pm 8.8$ & 0.267 \\
\hline LDL-cholesterol (mg/dl) & $120[95.2-190.1]$ & $133.1[103.8-198]$ & $108.8[90.6-189]$ & $0.019 *$ \\
\hline Triglycerides $(\mathrm{mg} / \mathrm{dl})$ & 153 [111-199] & 149 [107.5-199.3] & 159 [116-198] & 0.496 \\
\hline$C R P(m g / L)$ & $2.19 \pm 1.43$ & $2.42 \pm 1.44$ & $1.99 \pm 1.39$ & $0.043 *$ \\
\hline Interleukin-6 $(\mathrm{pg} / \mathrm{ml})$ & $1.92 \pm 0.866$ & $2.31 \pm 0.932$ & $1.59 \pm 0.643$ & $<0.001 *$ \\
\hline$O x L D L(U / L)$ & $188.8 \pm 53.9$ & $190.0 \pm 54.7$ & $187.8 \pm 53.5$ & 0.784 \\
\hline \multicolumn{5}{|c|}{ ECG data and Echo measurements } \\
\hline LVH on ECG & $52(29.4 \%)$ & $20(24.4 \%)$ & $32(33.7 \%)$ & 0.235 \\
\hline Fractional shortening (\%) & $35[32-38]$ & $35.5[33-39]$ & 35 [32-37] & 0.121 \\
\hline Ejection fraction $(\%)$ & $65.7 \pm 6.6$ & $66.1 \pm 6.7$ & $65.3 \pm 6.5$ & 0.384 \\
\hline $\operatorname{LVMI}\left(\mathrm{g} / \mathrm{m}^{2}\right)$ & $58.0 \pm 18.8$ & $56.9 \pm 17.2$ & $58.9 \pm 20.2$ & 0.479 \\
\hline \multicolumn{5}{|l|}{ Endothelial measurements } \\
\hline$F M D(\%)$ & $9.9 \pm 7.2$ & $6.7 \pm 4.6$ & $12.6 \pm 7.9$ & $<0.001 * *$ \\
\hline$N M D(\%)$ & $13.4 \pm 5.7$ & $12.8 \pm 5.7$ & $13.9 \pm 5.6$ & 0.164 \\
\hline
\end{tabular}

Values are mean $\pm S D$, median [interquartile range] or number of subjects (\%).

Statistical significance at $* p<0.05$ and $* * p<0.001$.

CAC, coronary artery calcification; ACEIs, angiotensin-converting enzyme inhibitors; ARBs, angiotensin-II receptor blockers; CAD, coronary artery disease; BMI, body mass index; FRS, Framingham Study risk score; ABP, arterial blood pressure; HDL, high-density lipoprotein; LDL, low-density lipoprotein; CRP, C-reactive protein; OxLDL, oxidized low-density lipoprotein; ECG, electrocardiography; Echo, echocardiography; LVH, left ventricular hypertrophy; LVMI, left ventricular mass index; FMD, flow-mediated dilatation; NMD, nitroglycerin-mediated dilatation.

pixels with a CT density threshold of 130 Hounsfield units. CACS was calculated by multiplying the area of CAC by a factor rated 1-4 dictated by the maximum CT density within that lesion. CACS values were grouped into 4 ascending CAC grades: 0 (absent; rating =0), 1-99 (mild; rating =1), $100-399$ (moderate; rating $=2$ ) and $\geq 400$ (severe; rating $=3$ ). For each patient, CACS can be calculated for each of the major coronary arteries: left main, left anterior descending, left circumflex, and right coronary arteries; the sum of these gives the total CACS.

\section{Statistical Analysis}

Normality of distribution of the different variables was assessed by the 1-sample Kolmogorov-Smirnov test. The distribution of CACS was markedly skewed, because about half of the study participants had a CACS of 0; as such, it was resistant to normalization by $\log$ transformation and other techniques. Therefore, the CACS values were grouped into a dichotomous variable (ie, presence of any calcification or total absence of CAC) for use in logistic regression. Comparisons of means and medians of continuous data across a factor with 2 levels were done by Student's t-test and the Mann-Whitney U-test for normal and skewed data, respectively. Comparisons of group means across CAC grades were done by 1-way analysis of variance (ANOVA), with post-hoc tests (Scheffe or Games-Howell). Comparisons of proportions were done by the $x^{2}$ test. The correlations between CACS and the continuous variables' values were assessed by the Spearman test. A 2-sided significance level of 0.05 was used for all analyses, which were con- 
Table 2 Mean Distribution of Continuous Variables Across Ordinal CAC Grades

\begin{tabular}{|c|c|c|c|c|c|c|}
\hline & \multicolumn{4}{|c|}{ CAC grade } & \multirow[b]{2}{*}{$\begin{array}{c}p \text { value } \\
\text { (ANOVA) }\end{array}$} & \multirow[b]{2}{*}{$\begin{array}{l}p \text {-for- } \\
\text { trend }\end{array}$} \\
\hline & $\begin{array}{c}\text { Absent } \\
{[C A C S=0]} \\
(n=95)\end{array}$ & $\begin{array}{c}\text { Mild } \\
\text { [CACS }=1-99] \\
(n=34)\end{array}$ & $\begin{array}{c}\text { Moderate } \\
\text { [CACS }=100-399] \\
(n=33)\end{array}$ & $\begin{array}{c}\text { Severe } \\
{[C A C S \geq 400]} \\
(n=15)\end{array}$ & & \\
\hline Age (years) & $48.7 \pm 6.1^{a, b}$ & $51.4 \pm 5.4$ & $52.2 \pm 4.7$ & $54.7 \pm 4.9$ & $<0.001 * *$ & $<0.001 * *$ \\
\hline$F M D(\%)$ & $12.6 \pm 7.9^{a, b, c}$ & $8.5 \pm 4.8^{a}$ & $6.1 \pm 4.3$ & $4.2 \pm 3.6$ & $<0.001 * *$ & $<0.001 * *$ \\
\hline Interleukin-6 $(\mathrm{pg} / \mathrm{ml})$ & $1.59 \pm 0.64^{a, b, c}$ & $2.08 \pm 0.71$ & $2.34 \pm 1.01$ & $2.73 \pm 1.10$ & $<0.001 * *$ & $<0.001^{* *}$ \\
\hline$C R P(m g / L)$ & $1.99 \pm 1.39$ & $2.61 \pm 1.68$ & $2.18 \pm 1.19$ & $2.53 \pm 1.38$ & 0.125 & 0.129 \\
\hline$O x L D L(U / L)$ & $187.8 \pm 53.5$ & $191.2 \pm 57.7$ & $182.5 \pm 50.9$ & $203.9 \pm 56.4$ & 0.631 & 0.641 \\
\hline$N M D(\%)$ & $13.9 \pm 5.6$ & $13.5 \pm 6.8$ & $12.1 \pm 4.6$ & $12.7 \pm 5.3$ & 0.408 & 0.127 \\
\hline
\end{tabular}

${ }^{a} p<0.05$ vs severe coronary calcification group; ${ }^{b} p<0.05$ vs moderate coronary calcification group; ${ }^{c} p<0.05$ vs mild coronary calcification group.

Statistical significance at $* * p<0.001$.

ANOVA, analysis of variance; CACS, CAC score. Other abbreviations as in Table 1.

ducted using SPSS 15 for Windows (SPSS Inc, Chicago, IL, USA).

\section{Results}

We studied 177 subjects (103 males, 74 females) aged $40-59$ years. CAC was detected in 82 subjects $(46 \%$ of sample: 52 males, 30 females). In the CAC-positive group, the CACS range was 10-1,901 Agatston units (median [interquartile range] of 143 [31-311.25] units). The median CACS was higher in males than in females (2 [0-202] vs 1 [0-97.5] units), but the difference was not significant ( $\mathrm{p}=$ 0.117). Baseline characteristics and comparisons of subjects with and without CAC are shown in Table 1. The CACpositive group showed significantly higher means (medians) for age, 10-year FRS, LDL-cholesterol, CRP, and IL-6 level than the CAC-negative group, but significantly lower mean FMD.

Variance analysis (Table2) revealed significant differences in the mean distribution of age, serum IL-6 levels, and FMD percentage values across CAC grades, reflecting the possible associations of these variables with the increment of CACS categories. The differences in mean serum CRP levels, NMD percentage values, and OxLDL levels were insignificant. Obviously, we found a significant trend with increasing mean age and IL-6 level, and a reciprocal decremental trend of FMD as the CAC grade increased (pfor-trend $<0.001$ ).

After adjusting for gender and body mass index, significant positive correlations were found between $\log$ (CACS + 1) values and age $(r=0.401 ; p<0.001), 10$-year FRS ( $r=$ $0.453 ; p=0.002)$ [Fig 1A] and serum IL-6 levels $(r=0.442$; $\mathrm{p}<0.001)$ [Fig 1B]. On the other hand, CACS correlated negatively with FMD $(r=-0.511 ; \mathrm{p}<0.001)$ [Fig 1C]. CACS correlations with serum CRP levels $(r=0.111, p=0.221)$ [Fig 1D], OxLDL levels $(r=0.013, p=0.866)$ [Fig 1E], NMD values $(\mathrm{r}=-0.124 ; \mathrm{p}=0.203)[\mathrm{Fig} 1 \mathrm{~F}]$ and the other continuous variables were insignificant. The correlations between FMD values and the biomarkers (adjusted for age, gender, and body mass index) were insignificant $(\mathrm{r}=-0.058, \mathrm{p}=$ 0.444 for CRP; $r=-0.089, p=0.241$ for IL- $6 ; r=-0.093, p=$ 0.223 for OxLDL), and the same was found for the correlations of NMD values to these biomarkers $(r=0.011-0.049$; $\mathrm{p}=0.523-0.886$ ).

Following univariate logistic regression analysis for each independent variable against the CAC-positive outcome, the following were associated with CAC presence at $p \leq 0.1$ (the entry threshold), and were thus included in the final stepwise logistic regression model: age, 10-year FRS, LDLcholesterol, CRP, IL-6, and FMD. This model was also adjusted for the essential confounders (gender and body mass index). We found that age, IL-6 levels, and FMD percentage values were significantly and independently associated with the presence of CAC (Table 3 ). The final logistic regression model proved to be highly significant $(\mathrm{p}<0.001)$, with good calibration (Hosmer-Lemeshow Goodness-of-fit $\left.\mathrm{X}^{2}=6.33, \mathrm{p}=0.610\right)$ and high discriminative power (C-statistic $=0.85[0.80-0.91] ; p<0.001$, Nagelkerke $\left.R^{2}=0.45\right)$. The model's correlation matrix was carefully checked to avoid collinearity, which was excluded on the basis of reasonable correlations (generally, $\mathrm{r}< \pm 0.5$ ).

\section{Discussion}

CAC, which occurs exclusively in atherosclerotic arteries and is absent in normal ones, is an early step in the development of atherosclerosis. ${ }^{15}$ Quantification of CACS provides accurate assessment of the overall atherosclerotic plaque burden in the coronary tree; ${ }^{1}$ however, calcium does not concentrate exclusively at sites with severe coronary artery stenoses. ${ }^{16}$ There is no proven relationship between $\mathrm{CAC}$ and either vulnerable (complications-prone) plaque ${ }^{17}$ or the probability of plaque rupture ${ }^{18,19}$ However, pathology studies have shown that the extent of CAC within plaques tends to be related to the presence of healed plaque ruptures ${ }^{20}$ Moreover, vulnerable plaques tend to be those with less extensive calcium deposits and are frequently seen in a spotty distribution ${ }^{20}$ a finding supported by intravascular ultrasound studies of patients with acute coronary syndromes. ${ }^{21}$ In addition, a recent study that used spectral analysis of intravascular ultrasound radiofrequency data has reported an association between dense calcium deposition and culprit coronary atherosclerotic plaques with negative remodeling?2 Recently, Motoyama et al proposed spotty calcification as 1 of the triad CT features characterizing culprit lesions associated with acute coronary syndromes? ${ }^{23}$

The present study shows clearly the incremental value of studying CAC in the present, and the results suggest that it may have more widespread use for a variety of indications in the future. In fact, CAC scoring may be able to globally define a patient's CAD event risk by virtue of its strong association with total coronary atherosclerotic disease burden, as shown by correlation with pathologic specimens, ${ }^{1,24}$ Asymptomatic individuals with an intermediate FRS (between 10 and 20\% 10-year risk of estimated coronary events) may be reasonable candidates for CAD testing 

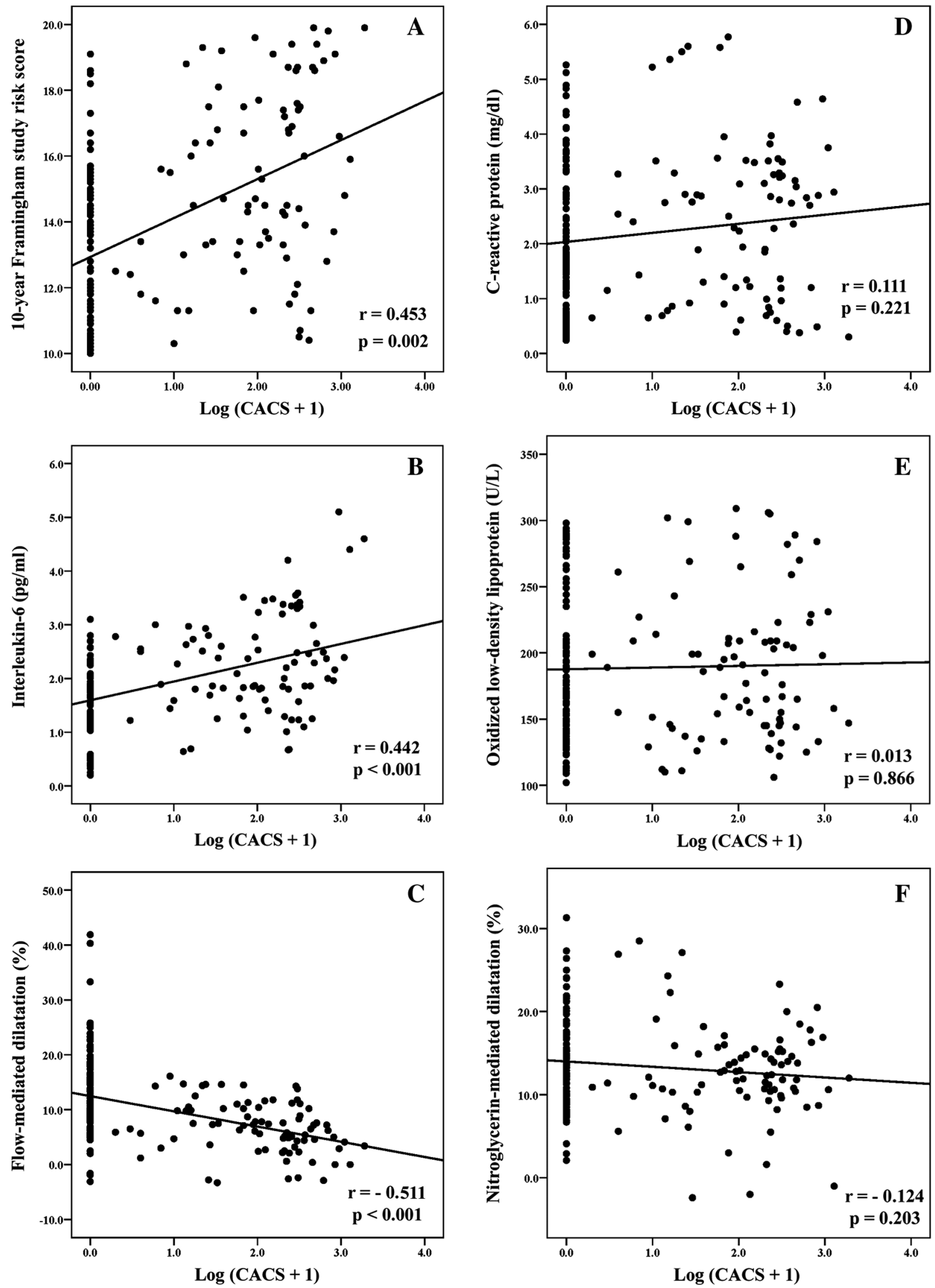

Fig 1. Scatter plots showing the correlations between $\log (\mathrm{CACS}+1)$ and 10-year Framingham Study risk score (A), interleukin-6 levels (B), flow-mediated dilatation percentage values (C), C-reactive protein levels (D), oxidized low-density lipoprotein levels (E), and nitroglycerin-mediated dilatation percentage values (F). CACS, coronary artery calcification score. 
Table 3 Results of Final Multivariate Logistic Regression Model Used to Test the Independent Association of the Input Variables With Positive Coronary Calcification Outcome

\begin{tabular}{lcccc}
\hline \hline & $\beta$-coefficient & OR & $95 \%$ CI for OR & $p$ value \\
\hline Age (years) & 0.079 & 1.083 & $1.014-1.156$ & $0.018^{*}$ \\
FMD $(\%)$ & -0.162 & 0.851 & $0.793-0.913$ & $<0.001^{* *}$ \\
Interleukin-6 $(\mathrm{pg} / \mathrm{ml})$ & 1.345 & 3.837 & $2.166-6.798$ & $<0.001^{* *}$ \\
10-year FRS & 0.022 & 1.022 & $0.975-1.071$ & 0.361 \\
LDL-cholesterol $(\mathrm{mg} / \mathrm{dl})$ & -0.003 & 0.997 & $0.988-1.005$ & 0.445 \\
CRP $(\mathrm{mg} / \mathrm{L})$ & 0.096 & 1.101 & $0.846-1.431$ & 0.476 \\
\hline
\end{tabular}

Statistical significance at $* p<0.05$ and $* * p<0.001$.

OR, odds ratio; CI, confidence interval. Other abbreviations as in Table 1.

using CAC as a potential means of modifying risk prediction and altering therapy? 25 This category of patients represents the population of particular interest for the purpose of primary prevention and risk identification and reduction. We found CAC in $46 \%$ of subjects in the aforementioned category. In addition, we found a significant moderate association between $\mathrm{CAC} /$ endothelial dysfunction and 1 (IL-6), but not the other (CRP, OxLDL), inflammatory markers we tested.

It has been reported that age and gender are the strongest clinical correlates of CAC ${ }^{26}$ Also, high CAC prevalence is reported in subjects $>60$ years regardless of the presence of risk factors ${ }^{27}$ In agreement those findings 26,27 we found an independent association between age and $\mathrm{CAC}$ even before the $7^{\text {th }}$ decade. The proportion of CAC-positive subjects rose steeply in the $6^{\text {th }}$ decade (56\%) compared with the $5^{\text {th }}$ $(34 \%)$ and $4^{\text {th }}(10 \%)$ decades (p-for-trend $\left.=0.030\right)$. In contrast to the other studies ${ }^{26,27}$ we did not find a significant influence of gender upon CAC presence.

To the best of our knowledge, only 2 prior studies have investigated the association of CAC (by electron-beam CT) with endothelial function in symptomatic ${ }^{5}$ and asymptomatic ${ }^{6}$ individuals along the entire CAD risk spectrum. Our results reveal a significant association between endothelial dysfunction (ie, reduced FMD) and CAC after multivariate adjustment, which is in accordance with the results of Huang et al, but contradictory to the findings of Kullo et al 6 The reasons for the discrepancies with the latter study are not fully clear; however, differences in study design, methods, and population characteristics may be possible factors. In our study, the lack of association between CACS and NMD (which reflects endothelium-independent dilatation via passive donation of nitric oxide), together with the emergence of FMD as an independent inverse correlate of CAC, signify a possible role of abnormal endothelium in the pathogenesis of CAC, which may involve pathways other than reduced nitric oxide release. In this respect, one might consider for future CAC studies using biomarkers that are more specifically linked with endothelial function, such as looking at the levels of $\mathrm{CD}^{34+} \mathrm{KDR}^{+}$endothelial progenitor cells $^{28}$ in peripheral blood in this category of patients. In fact, the use of this cellular marker would unify the complex interactions of multiple negative factors and may yield a better picture of in vivo mechanisms ${ }^{28}$ Budoff et $\mathrm{al}^{29}$ examined the association of CACS with brachial artery measures (distensibility and resistance) in 201 asymptomatic subjects $(70 \%$ men) referred by physicians for risk evaluation using electron-beam CT scans. They demonstrated a fair correlation between decreasing brachial artery measurements and increasing CACS. However, that study did not evaluate FMD, NMD, or inflammatory markers, so the results were not adjusted for these biomarkers, which might have had a significant influence.

Park et al found that CACSs and plasma CRP levels provided incremental information regarding the risk of clinical cardiovascular events in asymptomatic non-diabetic subjects ${ }^{30}$ In our study, we found a borderline significant association between CRP level and CAC presence per se, which failed to persist after multivariate adjustment, denoting a possible effect of the other confounders. Similar to our results, the Study of Inherited Risk of Coronary Atherosclerosis (SIRCA) documented a gender-specific association between CRP and CAC that was completely lost after adjustment for measures of adiposity 31 Although many studies have reported conflicting results about the relationship between CRP and CAC in asymptomatic individuals, the importance of the SIRCA study derives from its use of the largest sample set to date.

To our knowledge, only 1 study has evaluated the relationship between OxLDL and CACS in patients with CAD and documented a non-significant correlation 32 In our study, we investigated this relationship in asymptomatic subjects with a relatively larger sample size than that of Sherer et al 32 We did not find any significant association between OxLDL and CACS. Although the presence and magnitude of CAC is related to the level of LDL-cholesterol,33 it seems that the oxidation of LDL might not significantly participate in the process of CAC pathogenesis.

A novel result reported here is the association of IL-6 not only with CACS in the univariate and variance analyses, but also as an important independent correlate of CAC in the multivariate analysis. Thus, the possible role of IL-6 in the $\mathrm{CAD} / \mathrm{CAC}$ interaction matrix may be a reasonable rationale for its inclusion in cardiovascular risk prediction and therapeutic algorithms. However, to address why only IL-6, but neither CRP nor OxLDL, was associated with CAC, we must consider the mechanism of vascular calcification. It is now known that vascular calcification is a regulated process similar in many respects to bone formation 34 Also, cells with osteoblastic characteristics, termed "calcifying vascular cells", have been isolated from the vascular media:35,36 Well-documented findings suggest that differentiation of vascular cells plays a pivotal role by proceeding along osteogenic and chondrogenic lineages, resulting in calcified vascular tissues 35,37 One may hypothesize that an increase of IL-6 level would induce the latter process, whereas a similar induction by CRP or OxLDL may not be sufficiently strong in asymptomatic individuals with subclinical atherosclerosis.

The present findings support the utility of including information on endothelial function and serum IL-6 level in current methods of global risk assessment and in practice guidelines for asymptomatic patients in the intermediaterisk category of the 10-year FRS for CAD events. Measures 
to improve endothelial function and reduce the IL-6 level (ie, treatment with aspirin, statins and angiotensin-converting enzyme inhibitors ${ }^{38}$ ) might be of value in preventing $\mathrm{CAC}$ development, or arresting its progression, in these patients.

\section{Study Limitations}

The cross-sectional nature of this study makes it difficult to infer causality between CAC and endothelial dysfunction/inflammatory markers. Also, issues of selection or referral bias are likely when using a clinical cohort, as in our study, compared with population samples. Third, the ability to generalize these results to other ethnicities may not be appropriate. Finally, in addition to using an earlier version of the Framingham risk equation, there are no published data regarding the application of FRS to Middle Eastern populations. However, the FRS instrument has been validated in racially diverse populations, including the Atherosclerosis Risk in Communities (ARIC) and Cardiovascular Health Studies (CHS) with reasonable agreement between predicted and actual event rates in these population-based cohorts, making recalibration of the equations unnecessary 39,40

\section{Conclusions}

IL-6, but neither CRP nor OxLDL, was independently associated with CAC presence, whereas FMD was an independent inverse correlate of CAC. Testing for endothelial function and serum IL-6 level may facilitate cardiovascular risk assessment and improve therapeutic guidelines in asymptomatic patients at intermediate risk for CAD.

\section{Acknowledgment}

Mahmoud M. Ramadan, MD, was supported by a research grant from the Japanese Ministry of Education, Culture, Sports, Science and Technology, Tokyo, Japan.

\section{References}

1. Rumberger JA, Simons DB, Fitzpatrick LA, Sheedy PF, Schwartz RS. Coronary artery calcium area by electron-beam computed tomography and coronary atherosclerotic plaque area: A histopathologic correlative study. Circulation 1995; 92: 2157-2162.

2. Berliner JA, Navab M, Fogelman AM, Frank JS, Demer LL, Edwards PA, et al. Atherosclerosis: Basic mechanisms: Oxidation, inflammation, and genetics. Circulation 1995; 91: 2488-2496.

3. Halcox JPJ, Schenke WH, Zalos G, Mincemoyer R, Prasad A, Waclawiw MA, et al. Prognostic value of coronary vascular endothelial dysfunction. Circulation 2002; 106: 653-658.

4. Heitzer T, Schlinzig T, Krohn K, Meinertz T, Munzel T. Endothelial dysfunction, oxidative stress, and risk of cardiovascular events in patients with coronary artery disease. Circulation 2001; 104: $2673-$ 2678.

5. Huang PH, Chen LC, Leu HB, Ding PY, Chen JW, Wu TC, et al. Enhanced coronary calcification determined by electron beam CT is strongly related to endothelial dysfunction in patients with suspected coronary artery disease. Chest 2005; 128: 810-815.

6. Kullo IJ, Malik AR, Bielak LF, Sheedy PF 2nd, Turner ST, Peyser PA. Brachial artery diameter and vasodilator response to nitroglycerine, but not flow-mediated dilatation, are associated with the presence and quantity of coronary artery calcium in asymptomatic adults. Clin Sci (Lond) 2007; 112: 175-182.

7. Kritchevsky SB, Cesari M, Pahor M. Inflammatory markers and cardiovascular health in older adults. Cardiovasc Res 2005; 66: $265-$ 275.

8. Hansson GK. Inflammation, atherosclerosis, and coronary artery disease. $N$ Engl J Med 2005; 352: $1685-1695$.

9. Witztum JL, Steinberg D. Role of oxidized low-density lipoprotein in atherogenesis. J Clin Invest 1991; 73: 155-167.

10. Anderson K, Wilson P, Odell P, Kannel W. An updated coronary risk profile. Circulation 1991; 83: 356-362.

11. Kotani K, Maekawa M, Kanno T, Kondo A, Toda N, Manabe M. Distribution of immunoreactive malondialdehyde-modified low-density lipoprotein in human serum. Biochem Biophys Acta 1994; 1215: $111-118$.

12. Corretti MC, Anderson TJ, Benjamin EJ, Celermajer D, Charbonneau F, Creager MA, et al. Guidelines for the ultrasound assessment of endothelial-dependent flow-mediated vasodilation of the brachial artery: A report of the International Brachial Artery Reactivity Task Force. J Am Coll Cardiol 2002; 39: 257-265.

13. Soga J, Nishioka K, Nakamura S, Umemura T, Jitsuiki D, Hidaka T, et al. Measurement of flow-mediated vasodilation of the brachial artery: A comparison of measurements in the seated and supine positions. Circ J 2007; 71: 736-740.

14. Agatston AS, Janowitz WR, Hildner FJ, Zusmer NR, Viamonte M Jr, Detrano R. Quantification of coronary artery calcium using ultrafast computed tomography. J Am Coll Cardiol 1990; 15: 827-832.

15. Simons DB, Schwartz RS, Edwards WD, Sheedy PF, Breen JF, Rumberger JA. Noninvasive definition of anatomic coronary artery disease by ultrafast computed tomographic scanning: A quantitative pathologic comparison study. J Am Coll Cardiol 1992; 20: $1118-$ 1126.

16. Beckman JA, Ganz J, Creager MA, Ganz P, Kinlay S. Relationship of clinical presentation and calcification of culprit coronary artery stenoses. Arterioscler Thromb Vasc Biol 2001; 21: 1618-1622.

17. Davies MJ. The composition of coronary artery plaque. N Engl J Med 1993; 69: 377-381.

18. Falk E, Shah PK, Fuster V. Coronary plaque disruption. Circulation 1995; 92: 657-671.

19. Fuster V, Lewis A. Conner Memorial Lecture: Mechanisms leading to myocardial infarction: Insights from studies of vascular biology. Circulation 1994; 90: 2126-2146.

20. Burke AP, Taylor A, Farb A, Malcom GT, Virmani R. Coronary calcification: Insights from sudden coronary death victims. $Z$ Kardiol 2000; 89(Suppl 2): 49-53.

21. Ehara S, Kobayashi Y, Yoshiyama M, Shimada K, Shimada Y, Fukuda D, et al. Spotty calcification typifies the culprit plaque in patients with acute myocardial infarction: An intravascular ultrasound study. Circulation 2004; 110: 3424-3429.

22. Higashikuni Y, Tanabe K, Yamamoto H, Aoki J, Nakazawa G, Onuma $\mathrm{Y}$, et al. Relationship between coronary artery remodeling and plaque composition in culprit lesions: An intravascular ultrasound radiofrequency analysis. Circ J 2007; 71: 654-660.

23. Motoyama S, Kondo T, Sarai M, Sugiura A, Harigaya H, Sato T, et al. Multislice computed tomographic characteristics of coronary lesions in acute coronary syndromes. J Am Coll Cardiol 2007; 50: 319-326.

24. O'Rourke RA, Brundage BH, Froelicher VF, Greenland P, Grundy SM, Hachamovitch R, et al. American College of Cardiolo-gy/ American Heart Association Expert Consensus Document on electron-beam computed tomography for the diagnosis and prognosis of coronary artery disease. J Am Coll Cardiol 2000; 36: 326-340.

25. Greenland P, Bonow RO, Brundage BH, Budoff MJ, Eisenberg MJ, Grundy SM, et al. CCF/AHA 2007 clinical expert consensus document on coronary artery calcium scoring by computed tomography in global cardiovascular risk assessment and in evaluation of patients with chest pain: A report of the American College of Cardiology Foundation Clinical Expert Consensus Task Force (ACCF/AHA Writing Committee to Update the 2000 Expert Consensus Document on Electron Beam Computed Tomography). Circulation 2007; 115: $402-426$.

26. Allison MA, Wright CM. Age and gender are the strongest clinical correlates of prevalent coronary calcification (R1). Int J Cardiol 2005; 98: $325-330$.

27. Wong ND, Kouwabunpat D, Vo AN, Detrano RC, Eisenberg H, Goel $\mathrm{M}$, et al. Coronary calcium and atherosclerosis by ultrafast computed tomography in asymptomatic men and women: Relation to age and risk factors. Am Heart J 1994; 127: 422-430.

28. Werner N, Kosiol S, Schiegl T, Ahlers P, Walenta K, Link A, et al. Circulating endothelial progenitor cells and cardiovascular outcomes. N Engl J Med 2005; 353: 999-1007.

29. Budoff MJ, Flores F, Tsai J, Frandsen T, Yamamoto H, Takasu J. Measures of brachial artery distensibility in relation to coronary calcification. Am J Hypertens 2003; 16: 350-355.

30. Park R, Detrano R, Xiang M, Fu P, Ibrahim Y, LaBree L, et al. Combined use of computed tomography coronary calcium score and Creactive protein levels in predicting cardiovascular events in nondiabetic individuals. Circulation 2002; 106: 2073-2077.

31. Reilly MP, Wolfe ML, Localio AR, Rader DJ. C-reactive protein and coronary artery calcification: The Study of Inherited Risk of Coronary Atherosclerosis (SIRCA). Arterioscler Thromb Vasc Biol 2003; 
23: $1851-1856$.

32. Sherer Y, Tenenbaum A, Praprotnik S, Shemesh J, Blank M, Fisman EZ, et al. Coronary artery disease but not coronary calcification is associated with elevated levels of cardiolipin, beta-2-glycoprotein-I, and oxidized LDL antibodies. Cardiology 2001; 95: 20-24.

33. Allison MA, Wright CM. A comparison of HDL and LDL cholesterol for prevalent coronary calcification. Int J Cardiol 2004; 95: 55-60.

34. Boström K. Insights into the mechanism of vascular calcification. Am J Cardiol 2001; 88: 20E-22E.

35. Boström KI. Cell differentiation in vascular calcification. Z Kardiol 2000; 89(Suppl 2): 69-74.

36. Boström K, Watson KE, Horn S, Wortham C, Herman IM, Demer LL. Bone morphogenetic protein expression in human atherosclerotic lesions. J Clin Invest 1993; 91: 1800-1809.
37. Boström K, Demer LL. Regulatory mechanisms in vascular calcification. Crit Rev Eukaryot Gene Expr 2000; 10: 151-158.

38. Schieffer B, Drexler H. Role of 3-hydroxy-3-methylglutaryl coenzyme a reductase inhibitors, angiotensin-converting enzyme inhibitors, cyclooxygenase- 2 inhibitors, and aspirin in anti-inflammatory and immunomodulatory treatment of cardiovascular diseases. Am J Cardiol 2003; 91: 12H-18H.

39. D'Agostino RB Sr, Grundy S, Sullivan LM, Wilson P; CHD Risk Prediction Group. Validation of the Framingham coronary heart disease prediction scores: Results of a multiple ethnic groups investigation. JAMA 2001; 286: 180-187.

40. Weiner DE, Tighiouart H, Griffith JL, Elsayed E, Levey AS, Salem $\mathrm{DN}$, et al. Kidney disease, Framingham risk scores, and cardiac and mortality outcomes. Am J Med 2007; 120: 552.e1-552.e8. 